\section{TRUMP, FERNSEHEN UND DAS NEUE GAMP}

Einen Monat nach der Wahl Donald Trumps zum 45. Präsidenten der Vereinigten Staaten ließ das Time Magazine verlauten, «Donald Trump ist der erste wahre Reality-TV-Präsident». ${ }^{1}$ In dem Artikel, geschrieben von niemand Geringerem als dem Kommunikationsdirektor des Ex-Vizepräsidenten Dan Quayle (der selbst Probleme mit dem Image hatte, als intellektuell ungeeignet zu gelten), argumentiert Jeff Nesbit, das Weiße Haus würde bald als Setting für eine politische Realityshow dienen: «Jeder Tag gerät buchstäblich zu einer neuen Episode, in Echtzeit gedreht, vor einem Publikum und einer Welt, die nicht genug bekommt von dem Spektakel». ${ }^{2}$ Die Worte sollten sich als prophetisch erweisen: Ein Jahr später bekennen sich Menschen auf der ganzen Welt zu der halb entsetzten Obsession mit den täglichen Trump-Neuigkeiten, während die Late-Night-Politsatiriker_innen in den USA mit der Berichterstattung über die Possen «eines Weißen Hauses ... verzehrt von belanglosen Dramen» ${ }^{\mathbf{3}}$ eine internationale Zuschauer_innenschaft erreichen und sich eifrige Leser_innen für ein Exemplar von Michael Wolffs explosivem Buch Fire and Fury: Inside the Trump White House 4 reihenweise anstellen. Wie Nesbit vorausgesagt hat, sind die internen Machenschaften des Weißen Hauses die heißeste Show in der Stadt geworden - mit Trump im Mittelpunkt, das Rampenlicht an sich reißend.

Der Ausdruck <Reality-TV-Kandidat zirkulierte in Medien- und Wissenschaftskreisen bereits lange vor der Wahl 20I6, nahezu stets im Zusammenhang mit der Herabsetzung Trumps durch seine Verbindung mit dem, was wir als niederstes Genre des Fernsehens <kennen〉: Realitätsfernsehen. Kurioserweise gehörte Trump selbst zu denen, die die Meinung vertraten, Reality TV sei eher etwas für die <Widerlinge der Gesellschaft $>$, als ihm zum ersten Mal die Moderation von The Apprentice (NBC, 2004-20I7) angeboten wurde..$^{5}$ Aber was heißt es eigentlich, einen amerikanischen Präsidenten zu haben, dessen <Marke> nicht nur (wörtlich) an Türmen, Flugzeugen und gescheiterten Universitäten hängt, sondern auch mit dem Format und den Diskursen des Realitätsfernsehens verknüpft ist? Obgleich einige Beobachter_innen das Realitätsfernsehen
1 Dan Quayle: Donald Trump Is the First True Reality TV President, in: Time Magazine, dort datiert 9.12.2016, time.com/4596770/ donald-trump-reality-tu/, gesehen am 19.2.2018. Alle Übers. in diesem Text S. B.

2 Ebd.

3 Late Night with Seth Meyers, 14.12.2017.

4 Vgl. Michael Wolff: Fire and Fury: Inside the Trump White House, New York 2018.

5 Vgl. Mark Fisher: Donald Trump, Remade by Reality TV, in: The Washington Post, dort datiert 27.1.2016, www.washingtonpost. com/sf/national/2016/01/27/deciderstrump/?utm_term $=.883343 d_{52}$ cfc, gesehen am 18.2.2018. 

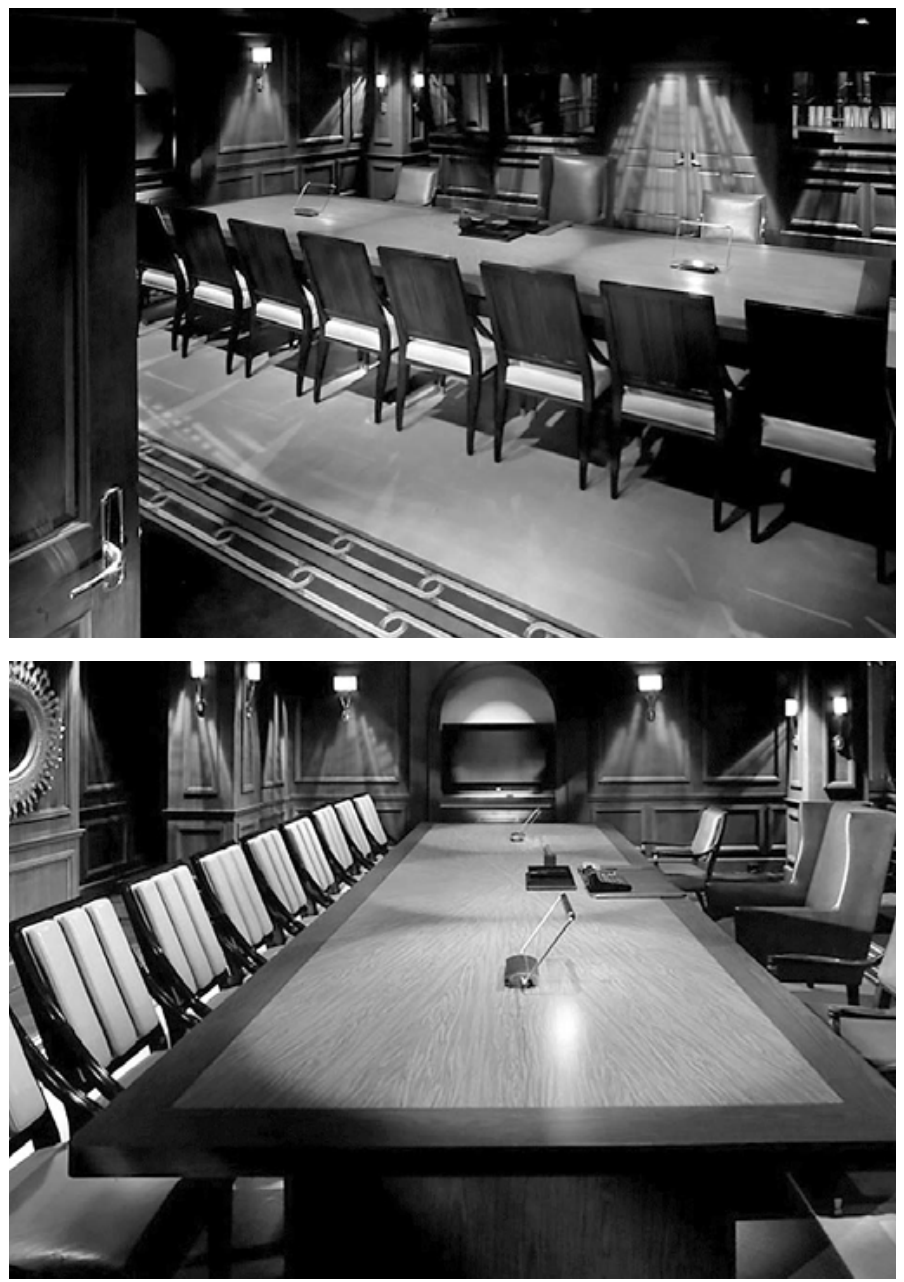

Abb. 1 Der Boardroom in der Reality-TV-Show The Apprentice

(NBC, 2004-2017) 
unmittelbar für Trumps Aufstieg verantwortlich machen, setzt diese Behauptung eine Verbindung zwischen populistischem Fernsehen und Regierungsämtern voraus, deren Erörterung unsere Aufgabe sein sollte. Schließlich wurde die US-Unterhaltungsindustrie, so bedeutend sie ist, zumindest auf einer ideologischen Trennung zwischen Hollywood und Washington gegründet - eine Trennung, die selbst Reagans Regierungszeit hindurch fortbestand, als man ihn von der Oberflächlichkeit der Westküste zur Wesentlichkeit des ColumbiaBezirks aufsteigen sah. Anstatt also verzweifelt unsere Köpfe zu schütteln, weil es unvermeidlich oder unmöglich - oder beides - sei, dass das Fernsehen einen Präsidenten hervorbringen könne, sollten wir fragen, was das Realitätsfernsehen im Besonderen ausmacht, das Trump den Weg zum Weißen Haus geebnet hat, und was die Implikationen dieser beunruhigenden Verbindung von Fernsehrealität und politischer Realität sind.

Der Ausdruck <Reality-TV-Präsident> bietet einen geeigneten Ausgangspunkt für diese Fragestellung, weil er auf mehreren Ebenen operiert. Im wörtlichen Sinne ist Trump ein <Reality-TV-Präsident〉, schlicht weil er zum Zeitpunkt seiner Amtseinführung am 20. Januar 2017 in beide Aktivitäten involviert war. Obwohl Trump von seiner Bildschirmrolle als autoritärer Moderator sowie Dreh- und Angelpunkt von The Apprentice zurücktrat, als er seinen Anspruch auf die Präsidentschaft anmeldete, blieb er einer der Executive Producer der fünfzehnten Staffel, die in den USA von Anfang Januar bis Mitte Februar 2017 ausgestrahlt wurde. ${ }^{6}$ Er behielt zudem einen großen Anteil an der Sendung; laut seines eigenen prahlerischen Tweets vom I6. Dezember 2016 mutmaßlich inklusive einer finanziellen Beteiligung als Executive Producer. Das mag erklären, warum Trump, direkt nach der Amtseinführung, einen Twitter-Krieg mit seinem Apprentice-Nachfolger Arnold Schwarzenegger (selbst bekannt für die Überwindung der Kluft zwischen Unterhaltung und Regierung) anzettelte, in dem er über dessen schlechte Performance spottete und ihn für die schlechten Quoten verantwortlich machte. Zuletzt lachen sollte allerdings Kaliforniens Ex-Gouverneur Schwarzenegger, als er suggerierte, dass es der Welt besser ginge, wenn die beiden ihre Rollen tauschten, und mithin implizierte, dass die Reality-TV-Show die Grenzen von Trumps Fähigkeiten markiere. Nichtsdestotrotz positionierte der Twitter-Austausch den <Reality-TV-Präsidenten> als TV-Executive-Producer (dem Quoten wichtig sind), als abgesetzten RealityTV-Star (dem sein Ansehen wichtig ist), als Reality-TV-Zuschauer (dem Unterhaltung wichtig ist) und als amtierenden US-Präsidenten - alles in einem. In anderen Worten: Trump lancierte seine Präsidentschaftskarriere verstrickt in eine komplizierte Beziehung mit jenem TV-Genre, das ihn durch erfolgreiche Wahlkampfveranstaltungen ins Weiße Haus brachte.

Auf einer allgemeineren Ebene wirft der Übergang von Reality TV zur Präsidentschaft die Frage auf, was Trump in den elf Jahren und fünfzehn Staffeln von The Apprentice, in denen er in den Augen der amerikanischen Öffentlichkeit gleichbedeutend wurde mit der Show, gelernt hat. The Apprentice, konzipiert
6 Obwohl sich NBC im Juni 2015 von Trump trennte und bekannt gab, dass «[a]ufgrund der jüngsten abfälligen Bemerkungen Donald Trumps über Immigranten, NBC Universal sein Geschäftsverhältnis mit Herrn Trump beendet", wurde für die Rückkehr von The Celebrity Apprentice auf den Bildschirm 2017 die Produktionsfirma MGM beauftragt, die Trump als Executive Producer behielt. Vgl. Nolan Feeney: NBC Cuts Ties With Donald Trump Over Immigration Remarks, in: Time, dort datiert 29.6.2015, time. com/3940305/nbc-donald-trumpimmigration, gesehen am 18.2.2018, sowie Cynthia Littleton: Donald Trump to Remain Executive Producer on "Celebrity Apprentice», in: Variety, dort datiert 8.12.2016, variety. com/2016/tu/news/donald-trump-markburnett-celebrity-apprentice-executiveproducer-1201937420, gesehen am 18.2.2018. 
7 Vgl. Todd Spangler: Trump Plugs Supreme Court Nominee Announcement on Facebook Live, in: Variety, dort datiert 31.1.2017, variety.com/2017/digital/news/ trump-facebook-live-supreme-courtnominee-1201974710, gesehen am 18.2.2018.

8 Vgl. Marshall McLuhan: Understanding Media: The Extensions of Man, New York 1964.

9 Alison Hearn: Trumps ‘Reality) Hustle, in: Television \& New Media, Vol. 17, Nr. 7, 2016, 656-659, hier 657 .

10 Man denke etwa an die sehr öffentlichen Entlassungen zahlreicher Mitarbeiter_innen, von Michael Flynn über James Comey, Reince Priebus, Sean Spicer und, in einer der bisher ironischsten Entlassungen überhaupt, Omarosa ManigaultNewman, die Trump zum ersten Mal begegnete, als sie Kandidatin in der ersten Staffel von The Apprentice war und von ihm in Episode 9 gefeuert wurde. und produziert von Mark Burnett, dessen Reality-TV-Leumund darin besteht, die US-TV-Landschaft durch die Einführung von Survivor (CBS, seit 2000) verändert zu haben, ist ein Challenge-basierter Wettbewerb für Unternehmeraspirant_innen und daher eine Studioversion von Trumps eigenem Boardroom. Die Parallelen zwischen dem Fernseh-Boardroom-Verhalten und dem Benehmen im Weißen Haus sind leicht zu finden. Die Schnellfeuer-Entscheidungen in Trumps ersten dreißig Tagen im Amt, vor allem das ohne Beratung mit anderen Regierungsebenen angeordnete Reiseverbot, erinnern an die unberechenbare, sekundenschnelle Entscheidungsfindung hinter den verschlossenen Türen des The-Apprentice-Boardrooms. Ergötzte sich die Kinematografie der Show an langen Einstellungen auf Türen und schließende Aufzüge im Trump Tower, öffnet sich die Tür zum Oval Office regelmäßig, um sendefähige Momente preiszugeben (wie etwa die Unterzeichnung von Präsidentenverordnungen oder die Bekanntmachung der Nominierten für den Obersten Gerichtshof, die als Berufungsfernsehen gehypt und vom Kabelfernsehen bis hin zu Facebook Live weithin übertragen wurde ${ }^{7}$ ). Der unaufhaltsame Twitter-Account hat obendrein jene Rolle der Behind-the-scenes-TV-Kameras übernommen, die so wichtig für die Zwiebelschalen-Formate des Realitätsfernsehens ist; alldieweil @realDonaldTrump täglich oder stündlich Texte abfeuert, die <zeigen>, was <wirklich> in Donalds Kopf vorgeht. Trotz seines Kampfes mit <den Medien > - ein Begriff, den er synonym mit <Fake News $>$ verwendet, im Gegensatz zu seiner eigenen vermeintlich unmittelbaren Authentizität -, ist Trump selbst eine Art Medium, in Marshall McLuhans Sinne, dass das Medium die Botschaft ist, was bedeutet, dass die Form soziopolitisch effektiver ist als der Inhalt. ${ }^{8}$ Mit dem Hinweis auf eine Studie, die zeigt, dass Trumps Kampagne 2015 von den großen Sendern mehr Sendezeit erhielt als alle anderen Kandidat_innen zusammen, bemerkte Alison Hearn während der Kampagnenmonate, dass die Vorwahlkandidat_innen «politische Kampagnen führten, während Trump die Medien führte». ${ }^{9}$ Mit dem Einzug der Trump'schen Medienmaschinerie in das Oval Office überrascht es kaum, dass die Berichterstattung über Neuigkeiten aus dem Weißen Haus genauso ambivalent fesselnd geworden ist wie eine suchtinduzierende Reality-Show. Im Hinblick auf dieses Regierungsfernsehen wird das durch die Tatsache unterstützt, dass die politische Unsicherheit von Trumps innerem Zirkel exakt das Format von The Apprentice reproduziert: Der Zwang, sich für Donalds Aufmerksamkeit gegenseitig zu bekämpfen, beschwört das Drama der Show im Oval Office - untermalt von der periodischen Deklaration «You're fired!» - mehr als Erfordernis des Mediums denn einer Meritokratie der individuellen Leistung. ${ }^{10}$ Mittlerweile brüstet sich Trump mit seinen Quoten - häufig wortwörtlich.

Wie sich zunehmend zeigt, ist Trump jedoch nicht nur ein Fernsehdarsteller und -produzent, sondern auch ein passionierter Fernsehzuschauer; zumindest von Kabelnachrichten und zweifellos des Senders Fox News. Wie John Cassidy im New Yorker schrieb, macht das Trump nicht nur zu einem 
Reality-TV-Präsidenten, sondern zu einem TV-Reality-Präsidenten - zu einem Staatsoberhaupt, gefangen in der Welt der Kabelnachrichten, wo jede Minute eine Eilmeldung generiert wird, jedes Thema relevant ist, jeder Schluckauf eine Krise und jede Kritik am Präsidenten, aus dessen eigener Sicht, eine Verleumdung darstellt. ${ }^{11}$ Fernsehen und Twitter sind in eine symbiotische Beziehung verstrickt: Trump schaut Fox, twittert darüber und Fox retwittert faktisch Trump. ${ }^{12}$ Beispielsweise führte Trumps Anstoß an der Behauptung, er schaue täglich vier bis acht Stunden Nachrichtenfernsehen, unlängst zu weiteren Tweets, über die ihrerseits in den Fernsehnachrichten berichtet wurde. ${ }^{13}$ Als erster durch und durch mediatisierter Präsident ist Trump mithin ein Produkt seiner Beziehungen zum Fernsehen auf beiden Seiten des Bildschirms: als Produzent von Reality-TV und als Konsument von Kabelnachrichten. Beide Genres operieren mit derselben Taktik überhöhten Dramas zur Entfaltung <des Realen>. Indem er über das Fernsehprogramm twittert, das er sich anschaut, erzeugt Trump tatsächlich die Realität; und indem er das tut, indem er also die Mechanismen des Regierens umgeht, die ansonsten die Realitätseffekte des Medialen beschränken, erweitert er einfach den performativen Effekt der Medien bis in den politischen Bereich.

Sofern Trumps überraschende Wahl eines bewiesen hat, dann, dass wir es uns weder erlauben können, das Realitätsfernsehen als <nicht real> abzutun, noch großherzig anzunehmen, dass der Reality-TV-Präsident die Politik mit der Fiktion infiziert habe. Im Gegenteil: Fernsehen ist das, was das Phänomen Trump für einen Großteil der amerikanischen Öffentlichkeit sebr real hat werden lassen - und zwar real auf eine bestimmte Weise, als Medium des Gefühls -, gerade durch seine Erfahrung mit dem und seiner Exponierung im Realitätsfernsehen. Sofern das Realitätsfernsehen durch seine konstruierten Fiktionen Anspruch auf das Reale erhebt, ist Trump gewissermaßen einer seiner realsten Effekte - oder besser: einer seiner realsten Affekte. Ein doppelter Widerspruch ist hier wirksam: Einerseits lässt sich die Hyperrealität des Fernsehens durch den Affekt von der sozialen und politischen Realität zunehmend schwerer unterscheiden, andererseits ist dieser Affekt selbst positiv und negativ gleichermaßen, was einen paradoxerweise lebensbejahenden Ausdruck negativer Emotionen hervorbringt. Rachel Dubrofsky hat auf Trumps widersprüchliche Position als präsidialer Milliardär mit vulgärem Geschmack und grobem Benehmen aufmerksam gemacht. ${ }^{14}$ Aber Selbstentblößung ist der Modus Operandi einer Reality-TV-Persönlichkeit, besonders dann, wenn diese EntblöBung schlechtes Benehmen in ausgesprochen gegenderten, klassifizierten, rassifizierten und celebritisierten Formen beinhaltet - wie etwa das berüchtigte Gezänk im Serienfranchise The Real Housewives auf Bravo (seit 2006) ${ }^{\mathbf{1 5}}$ oder eigene Maßregelungen im Sitzungssaal in The Apprentice. Mit seinen kleinen Händen, großen Türmen und der Vorliebe fürs Vergolden agiert Trump wie ein Fernsehspektakel des schlechten Benehmens. Obwohl der Reiz derartiger Sendungen besonders von der gebildeten, liberalen Elite oft als Beispiel für
11 Vgl. John Cassidy: Donald Trump is the «TV Reality» President, in: The New Yorker, dort datiert 6.2.2017, www.newyorker.com/news/ john-cassidy/donald-trump-is-thetu-reality-president, gesehen am 18.2.2018.

12 Vgl. Tom Kludt, Tal Yellin: Trump Tweets and the TV News Stories Behind Them, in: CNN Money, dort datiert 27.2.2017, money.cnn. com/interactive/media/trump-tu-tweets/ index.html, gesehen am 18.2.2018.

$13 \mathrm{Vgl}$. Andrew Griffin: Donald Trump Tweets to Deny Watching 8 Hours of TV a Day, amid Major Security Operation after Suspect New York Bomb Attack, in: Independent, dort datiert 11.12.2017, hdl. handle.net/11346/IKL4, gesehen am 18.2.2018.

14 Vgl. Rachel Dubrofsky: Authentic Trump, in: Television \& New Media, Vol. 17, Nr. 7, 2016, 663-666; Feeney: NBC Cuts Ties With Donald Trump.

15 Vgl. Pier Dominguez: «l'm Very Rich, Bitch!» The Melodramatic Money Shot and the Excess of Racialized Gendered Affect in the Real Housewives Docusoaps, in: Camera Obscura, Vol. 30, Nr. 1, 2015, 155-183. 
den kulturellen Untergang verteufelt wird, ist es exakt die Energie dieses televisuell kodierten Verhaltens, die Trump angezapft und in die politische Landschaft eingespeist hat.

Donald Trump versteht den paradoxen Einsatz dramatischer, negativer Affekte als Teil des Vergnügungsmechanismus, der die Leute fesselt und mithin politische Effekte zeitigt. Die Politik dieses Einsatzes negativer Affekte verschiebt die Bedingungen und Techniken des Realitätsfernsehens, die Trump allzu gut kennt. Das, was Trump Freude bereitet, färbt seine Performance als Präsidentschaftskandidat bzw. Präsident und steigert das Vergnügen seiner Unterstützer_innen. Jener ideologischen oder moralischen Basis beraubt, die die Republikanische Partei kennzeichnet, und getragen von einem übergroßen Narzissmus, erzeugt Trumps charismatische Persönlichkeit einen FeedbackLoop, der einen neuen affektiven Autoritarismus präsentiert und in Szene setzt. Dieser ist von faschistischen Vorläufern dadurch zu unterscheiden, dass er auf einen medialen Werkzeugkasten zurückgreift, der mit Affekt statt Ideologie handelt. Ich möchte an dieser Stelle Camp als Bezugsrahmen verwenden, mit dem sich begreifen lässt, wie Trump-als-Medium das Realitätsfernsehen mit Politik verschränkt. Während Camp eine Strategie ist, mit der QueerPerformer_innen seit langem naturalisierte Genderhierarchien untergraben, haben wir es nun mit einem neuen Modus transgressiven Verhaltens zu tun, da Realitätsfernsehen, Twitter und Kabelnachrichten jene belohnen, die bereit sind, sich vor den Kameras aufzuspielen. Im schlimmsten Fall handelt es sich bei diesem Verhalten um einen Faschismus-als-Affekt, dessen Thrill nicht einer queeren Ironie des Überschwangs, sondern einer Wut entspringt, die im Theater der Affekte als neue <authentische> Währung akzeptiert wird. Es ist dieses Bündel von Widersprüchen, das ich als neue Form von Camp bezeichnen werde. Vergnüglich und bedrohlich zugleich, hat es in Trump seinen Oberbefehlshaber gefunden.

\section{«The Apprentice»: Boardroom-Kämpfe und das Vergnügen des Publikums}

Obwohl Trump schon seit den I980er Jahren, als er erstmals regelmäßig in Talkshows und Fernsehinterviews (z. B. bei Tom Brokaw, Oprah Winfrey, David Letterman) auftrat und öffentlich seinen (Wider-)Willen diskutierte, seinen Präsidentschaftsanspruch anzumelden, hat The Apprentice entscheidend dabei geholfen, sein Profil auf die nationale Bühne zu heben. Im Laufe der i $980 e r$ Jahre erlangte er wachsende Bekanntheit als Immobilienmagnat in New York City, ein Ruf, den er mit dem Selbsthilfe-Bestseller The Art of the Deal (1987) festigte und der ihn in populistischen Geschäftskreisen berühmt gemacht hat.

16 Emily Nussbaum: The TV That Created Donald Trump, in: The New Yorker, dort datiert 31.7.2017, www.newyorker.com/magazinel 2017/07/31/the-tu-that-created-donaldtrump, gesehen am 18.2.2018.
Das war auch der Zeitraum, in dem Trump «sich im Fernsehen selbst spielte». ${ }^{16}$ In einer Reihe von Statistenrollen in Filmen, Miniserien und Sitcoms wurde sein Ruf als Tycoon auf diese Art selbstreflexiv in eine Pointe verwandelt. Die I 99oer Jahre indes brachten Pleiten seiner Besitztümer und das Scheitern 
seiner Ehe, an welchem Punkt er, wie Emily Nussbaum bemerkt, «begann, sich als Star neuer Genres neu zu erfinden: Realitätsfernsehen, Pro-Wrestling und Kabelnachrichten, insbesondere Fox News». ${ }^{17}$ Nichts davon hatte jedoch eine vergleichbare Wirkung wie The Apprentice, auch wenn Trump bereits eine von einer Plattform konvergierender Medien geschaffene öffentliche Figur war, als Produzent Mark Burnett 2002 an ihn herantrat. Indem er sich mit dem Realitätsfernsehen zusammenschloss, erlangte Trump nicht nur eine neue Art der Medienprominenz - eine, die gewöhnliche Leute berühmt und berühmte Leute gewöhnlich macht,$-^{18}$ sondern ebnete den Weg für eine neue, vom Realitätsfernsehen untrennbare Form der individualisierten Politiker_innenberühmtheit, die Politiker_innen zugleich als autoritär und zugänglich präsentiert.

The Apprentice war durch die Sichtbarkeit des Mainstreamfernsehens auch entscheidend für die Entwicklung der Trump-Marke. Strukturiert um geschäftliche Challenges bot The Apprentice als Sendung Trump unendliche Gelegenheiten zum Management seiner Marke und für Produktplatzierungen (z. B. Trump Ice, «das beste Tafelwasser»). Wirtschaftskommentator_innen haben darauf hingewiesen, dass Trump sich, nach den Konkursen im Kontext seiner Casinoanteile in den frühen I99oer Jahren, von den materiellen Risiken der Grundstückserschließung abgewendet hat, um stattdessen das immaterielle Kapital seiner Marken aufzubauen. Laut einem NBC-News-Exposé eröffnete die Sendung Trump unzählige Lizenzierungsmöglichkeiten «und bestätigte seine Strategie des Branding-statt-Bauen», ${ }^{19}$ eine Strategie, die Hand in Hand ging mit einer Show über Verkaufs- und Marketing-Wettkämpfe. The Apprentice diente mithin der Konvergenz der Kommerzialisierung seines Markennamens mit einer exponentiell wachsenden Medienprominenz. Tatsächlich bemerkte Trump in einem Fernsehinterview von 20I 2, als er eine Bewerbung um die Präsidentschaft ernsthaft in Betracht zog, dass die Kandidatur Opfer fordern würde: «Das ist eine große Sache für mich, einschließlich der Tatsache, dass ich, offen gesagt, eine der erfolgreichsten Shows im Fernsehen aufgeben müsste, was viel Geld ist und viel Prestige und viel Macht». Interessanterweise musste Trump 2012 noch den Verlust von Geld, Prestige und Macht des Fernsehens gegenüber dem Gewinn von Prestige und Macht durch eine Präsidentschaft abwägen. 2015 scheint er erkannt zu haben, dass es, anstatt zwischen beidem wählen zu müssen, eine verwertbare Kontinuität zwischen Realitätsfernsehen und Politik gibt.

Es lohnt sich, einen genaueren Blick auf The Apprentice zu werfen, dessen unerwartete Popularität in der ersten Staffel ihm 2004 Platz 7 aller USFernsehprogramme einbrachte und dessen Finale von über 28 Millionen $\mathrm{Zu}$ schauer_innen gesehen wurde. Die Sendung bedient sich des wohletablierten Rufs Trumps als Tycoon-fürs-Fernsehen, um ihr Hauptthema zu vermitteln: Geld - und die Ideologie des <Hart-Arbeitens, um viel Geld zu verdienen〉. Der Titelsong, ein Ohrwurm der O'Jays mit dem Titel For the love of money ist eigentlich eine Kritik an Habgier und der selbstzweckhaften Geldanhäufung, aber die Sendung zelebriert bereits im Vorspann das scheinbar offensichtliche
17 Ebd.

18 Vgl. Misha Kavka: Reality TV, Edinburgh 2012.

19 NBC News: 200os: ‘Apprentices Helps Donald Trump Finally Launch A White House Bid, online unter www.youtube.com/ watch?v=TSVPDrR8GyQ, dort datiert 6.7.2016, gesehen am 18.2.2018. 
Erstrebenswerte an Helikoptern, Limousinen und dem Highlife Manhattans mit Teasern des superreichen Lifestyles als Belohnung für die Kandidat_innen. Nach einem Konzept von Mark Burnett begann die Sendung als Transplantat von Burnetts höchst erfolgreicher Reality-Show Survivor in den Großstadtdschungel der Geschäftswelt. Wie in Survivor leben die Kandidat_innen unter einem Dach, werden aber in Teams/Stämme aufgeteilt; alle paar Tage müssen sie sich neuen Business-Challenges stellen (meistens im Bereich Marketing), jede angeführt von dem_der Projektmanager_in der Woche. Jede Challenge endet mit einer Bewertung im Boardroom, die zwangsläufig dazu führt, dass mindestens ein_e Kandidat_in mit Trumps Slogan «You're fired!» eliminiert wird. Nach Emily Nussbaums Beschreibung des Boardroom-Rituals im New Yorker, «endet jede Episode grandios, wenn Trump eintrifft, um das Urteil zu fällen. Zuerst analysiert das Verliererteam seine Versäumnisse. Dann kriechen drei Kandidat_innen vor ihm zu Kreuze. Schließlich wird eine_r gefeuert und macht im goldenen Aufzug den Abflug».20

Die Mise en Scène des Boardrooms selbst ist hier von besonderem Interesse (Abb. I). Die Kandidat_innen stellen sich dort nebeneinander am Tisch auf, gegenüber von Trump, der, flankiert von seinen beiden Berater_innen, im Zentrum in einem großen, roten Sessel sitzt. Es handelt sich ohne jeden Zweifel um ein TV-Setup (der Boardroom und die Wohnung der Kandidat_innen sind speziell angefertigte Studiobauten in einem Stockwerk des Trump Towers), der Mangel an <Realität〉 - im Sinne des tatsächlichen Aussehens eines Firmen-Boardrooms - ist jedoch unwichtig. Entscheidend ist vielmehr, dass diese Mise en Scène durch das Medium des Fernsehens erkennbarer, bekannter und realer wird als jeder echte Firmen-Boardroom, den tatsächlich nur sehr wenige Menschen zu sehen bekommen. Der Boardroom ist zudem nicht nur ein Ort autoritärer Entscheidungen (Trumps Wort ist in diesem Setting Gesetz und seine Berater_innen dürfen nur reden, wenn sie angesprochen werden), sondern auch von großer Affektivität - emotionale Höhen und Tiefen sind an dramatische Gipfelpunkte geknüpft und werden in den Ausscheidungs- oder eher Vernichtungssequenzen ausgespielt. Diese Höhepunkte erzeugen extreme Emotionen, gerade weil das Leben der Kandidat_innen dem Rhythmus Challenge - Boardroom - Belohnung/Scham unterliegt und sich ihr Selbstwertgefühl, nun über diesen Rhythmus definiert, auf Trumps Beurteilung ihrer Leistung reduziert. Da das Fernsehformat alles andere abgestreift hat, was ihr alltägliches Dasein ausmacht, sind Emotionen der Freude, Wut, Erleichterung, des Bedauerns oder Frust, die die Kandidat_innen durchleben, entsprechend verstärkt. Zusätzlich zu der materiellen Arbeit, die sie in den Challenges geleistet haben, meist im Auftrag von Episodensponsor_innen, leisten sie auch eine immaterielle affektive Arbeit ${ }^{21}$ - und erzeugen Emotionen, die wiederum

20 Nussbaum: The TV That Created Donald Trump.

$21 \mathrm{Vgl}$. Michael Hardt: Affective Labor, in: boundary 2, Vol. 26, Nr. 2, 1999, 89-100. den Wert des Programms steigern: für Zuschauer_innen, Sponsor_innen und für die Marke Trump. Zugleich sind die Kandidat_innen jedoch involviert in Business-Challenges, in denen sie sich mit Manager_innen, Klient_innen und 
Konsument_innen in der <realen Welt> auseinandersetzen müssen; entsprechend können wir nicht davon sprechen, dass die Realität von The Apprentice ganz und gar getrennt sei von der sozialen Realität. Was Zuschauer_innen lernen - und was Trump selbst von seinem Sessel in der Mitte des BoardroomTischs aus lernt -, hat mehr mit der affektiven Realität des Umgangs der Kandidat_innen miteinander zu tun als mit irgendeinem Geschäftstraining etwa im Sinne von MBA-Aufgaben oder The Art of the Deal.

Der Boardroom präsentiert Trump als ruhig, überlegt und stets überzeugt davon, die richtige Entscheidung getroffen zu haben. Obwohl er manchmal die Bestätigung seiner Berater_innen einholt, plagen ihn nie Zweifel bei einer schwierigen Entscheidung, noch stellt er das Format in Frage. Vielmehr ist er eine Funktion des Formats, das verlangt, dass jemand eliminiert wird, selbst wenn alle gleichwertige oder ausgeglichene Leistungen abgeliefert haben. Seine Fähigkeit, die <richtige> Entscheidung zu treffen, basiert auf einem Set an äußerlichen, oft widersprüchlichen und überwiegend willkürlichen Regeln wie z. B.: Stimme stets mit dem Chef überein, aber verschaffe deiner Stimme Gehör; übernimm Führungsverantwortung, aber tanze nicht aus der Reihe; tritt für dich ein, aber wisse, wann man den Mund zu halten hat, und sei auf keinen Fall <dumm> (was offenbar bedeutet: Errege nicht unnötig Trumps Aufmerksamkeit und bringe ihn nicht dazu, dich zu feuern). Der einzig konsistente Aspekt in diesem Training, das unter anderen Bedingungen in sich zusammenfallen würde, besteht darin, dass Trump immer Recht hat. Er ist das Gesetz dieser Sendung, der Meister-Signifikant ihrer Taktung, obwohl auch das die diskursive und affektive Aufladung des Formats ist. Regelmäßig verlaufen Trumps Entscheidungen anders, als wir, die scharfsinnigen Zuschauer_innen, es vorausgesehen haben. In vielen Fällen, gerade wenn es so aussieht, als ob eine bestimmte Person dabei ist, gefeuert zu werden, reißt Trump das Ruder herum, beginnt jemand anderen zu schikanieren, um schließlich eine weitere, vollkommen andere Person zu feuern. Oder Trump wechselt, wenn sich zwei Leute bekämpfen, um im Wettbewerb zu bleiben, plötzlich sein Augenmerk und findet Gründe, um eine wiederum andere Person zu feuern. Was hier geschieht, ist ein Prozess, der im Unterschied zu strategie- oder ergebnisorientierten Geschäftsentscheidungen der Kontingenz von affektiven Flows folgt. Trump selbst ist streng genommen nicht der Herr über diese Flows. Vielmehr ruht seine Position der Gewissheit auf einem ideologischen Vakuum, das von einer affektiven Intensität gefüllt ist, die das TV-Format produziert. Wie die Zuschauer_innen folgt auch Trump dem Affekt, klinkt sich an einer unerwarteten Stelle ein, um die Entscheidung, ex post facto oder nachträglich [deutsch i. Orig.; Anm. d. Ü.], als notwendig zu rechtfertigen. Woran es keinen Zweifel gibt und was die Linie zwischen Fernsehen und sozialer Realität durchlässig werden lässt, ist die Vertrautheit mit derartigen autoritären Wendungen in der realen Welt. Auch das Recht ist bekanntlich ein Tyrann. Es kann auf ebenso obszöne Weisen, ohne einvernehmliche oder vertragliche Grundlage, wirken. Trumps Gesetz ist sein 
22 YouTube-Titel für die derzeit zugängliche Version dieses Clips, veröffentlicht von TheEStream: National Debate Champ Gets Killed by a Bridal Salon Owner, www. youtube.com/watch?v=UAy54 Jtdubg, dort datiert 29.6.2011, gesehen am 18.2.2018.

23 Laut Kurt Andersen ist «der WWE, wenn nicht gar der Schlüssel, so doch ein wichtiger Schlüssel zum Phänomen Donald Trump, das wir gerade erleben", zit. n.: Eric Johnson: Full transcript: WNYC's Kurt Andersen on Recode Media, in: recode, dort datiert 1.11.2016, www. recode.net/2016/11/1/13480986/kurtandersen-wnyc-spy-donald-trumprecode-media-podcast-transcript, gesehen am 18.2.2018.
Wort, aber sein Wort basiert auf dem televisuellen Rhythmus, der durch formatgesteuerte Machtkämpfe und Entscheidungen affektive Flows generiert. In seinem Boardroom, real und unwirklich zugleich, herrscht weniger Trump als Drahtzieher als vielmehr der Affekt.

Obwohl es klar ist, dass Trump bei seinen Berater_innen Unterstützung sucht, um seine Entscheidungen nachträglich zu begründen - häufig mit den Worten, «das musste getan werden», die von beiden Seiten zustimmend abgenickt werden -, ist es weniger ersichtlich, dass er für seine Entscheidungen Deckung sucht, weil die Gründe für das Feuern eines Kandidaten oder einer Kandidatin immer nur augenscheinlich logisch sind. Tatsächlich sind die Entscheidungen das Ergebnis der sukzessive erhöhten Showtaktung, von der Vorstellung der Challenge über die Gruppendynamiken bis hin zu der übertriebenen, gefühlsgesteuerten Performance der Kandidat_innen im Boardroom. Trumps Rolle im Zusammenhang dieser affektiven Performances ist bemerkenswerterweise ebenfalls mehrdeutig, wenn man bedenkt, dass er, wie die Montage suggeriert, eine nachdenkliche, aber wachsame Distanz zum Gefühlsfeuerwerk bewahrt, aber gleichzeitig das Feuer schürt mit Fragen, die sich scheinheilig einlassen: «Lassen sie mich eine Frage stellen ...». In einer Boardroom-Auseinandersetzung aus der späten zweiten Staffel (Episode I2, NBC 20I4) entfacht Trump ein lautes Wortgefecht zwischen zwei Frauen, Jenny und Sandy, während der Projektmanager Andy, sicherer, aber viel jünger, dazwischen sitzt. Als die beiden Frauen anfangen, sich über den stammelnden Andy, der etwas zu sagen versucht, hinweg anzuschreien, besteht Trumps einzige Intervention in der Bemerkung, dass Andy, ein nationaler Debattiermeister, «ziemlich übel von Sandy zugerichtet wurde». ${ }^{22}$ In einem klassischen Apprentice-Akt des Spießumdrehens haut Trump schließlich auf den Tisch, richtet seinen Finger auf Andy und feuert ihn, weil er «geschlagen wurde». Das könnte ein Beispiel für die Boardroom-Logik sein, still zu sein und nicht die Aufmerksamkeit auf sich zu lenken. Aber in diesem Fall versagt die Regel: Andy wird gefeuert.

Wichtiger noch: Die Kandidat_innen, die einander anschreien, während sich Trump zurücklehnt und beobachtet, sind Frauen, was diese Auseinandersetzung in die Nähe einer diskursiven Form des Schlammringens bei einem Schönheitswettbewerb rückt - beides Unterhaltungsformen, die Trump durch seine Mitwirkung am Miss-Universe-Franchise und dem WWE (World Wrestling Entertainment) ${ }^{23}$ wohlbekannt sind. Ungeachtet seiner reglosen Miene, die (der Kamera) signalisiert, dass er eine starke Führungsperson ist, die zuhört, versucht Trump nicht, den Streit zu beenden, feuert ihn sogar an, indem er über Andy spottet, dass dieser seinem Ruf als Debattiermeister nicht gerecht werde. Gerade die Tatsache, dass das Schreiduell nicht im Entferntesten einer Diskussion ähnelt, liefert jedoch einen Hinweis darauf, warum der Streit ungehindert weiterlaufen kann: nämlich zu Trumps Freude und mithin zur Freude der Zuschauer_innen. Obwohl vielleicht nicht alle Zuschauer_innen Spaß am Frauenschlammringen finden, wörtlich oder metaphorisch, hat der aus den 
Fugen geratene Streit auf Sendung etwas Faszinierendes. Dieser Moment artikuliert die Sorte von qualvoll-erlesenem Vergnügen, die Lacan als jouissance bezeichnet und die sich hier, über die fehlende Logik von Trumps Entscheidungen, zu den affektiven Rhythmen des Realitätsfernsehens erweitert. Wie ich an anderer Stelle erörtert habe, ${ }^{24}$ feuerte Trump Andy nicht, weil er sich gegenüber den beiden Frauen nicht behaupten konnte, sondern, weil er selbst die Position des Beobachters einnahm, der sich am Frauenspektakel erfreut - eine Position, die zweifellos Donald selbst vorbehalten ist.

Bemerkenswert an dieser, mehr von der Kakophonie des affektiven Moments als von der simplen Identifikation mit Trump hypnotisierten jouissance des Zuschauens ist, dass wir es mit einer frühen Form desselben Vergnügens zu tun haben, das im Laufe weniger Jahre Zuschauer_innen von Bravo-RealityShows wie The Real Housewives und deren zahlreichen Spin-offs und Epigonen zur Verfügung stehen würde. Diese übertriebenen Performances beschränken sich in The Apprentice nicht auf Frauen, selbst wenn sie sich irgendwann in die in Bravo- und VHI-Shows stark favorisierten catfights verwandeln. Vielmehr tragen die Teilnehmer_innen von The Apprentice in ihrem Ansporn, für ihr Überleben zu kämpfen und bei Donald Trump gut dazustehen, dazu bei, das Realitätsfernsehen als Bildschirmschlachtfeld zu entwickeln, strukturell abhängig vom spektakularisierten Affekt. Im Zentrum dieser Formel und als Funktion dieses Medienformats sitzt Trump als Manifestation eines irrationalen, vom Affekt getragenen Gesetzes, das ihn als jene «obszöne Figur des Vergnügens» markiert, die Mark Andrejevic von Slavoj Žižek herleitet. Tatsächlich argumentiert Andrejevic, dass «Trumps Kampagne als die erste Reality-TV-Kandidatur beschrieben werden könnte, weniger wegen Trumps Status als Reality-TV-Berühmtheit, sondern weil er der für das Format charakteristischen Kombination von kluger Skepsis und unreflektierter Fantasie gerecht wird $\gg .{ }^{25}$ Diese Kombination von Skepsis und Fantasie ist Teil eines Umbruchs im Realitätsfernsehen hin zu einem wissenden Vergnügen an Pathos, Zurschaustellung und übertriebener Performance. Diese Performance umfasst zudem ein transgressives Verhalten, nunmehr sanktioniert nicht nur durch das Fernsehen, sondern auch auf der politischen Bühne.

\section{Das bedrohliche Antlitz von negativem Camp}

Zahlreiche Sendungen, vor allem, wenngleich nicht ausschließlich, des Reality-TV, ziehen ihren Reiz aus dem Spektakel schlechten Benehmens. Wie ich zeigen werde, erinnert das an das Spektakel des Camp, wenn auch nun seines progressiven politischen Potenzials beraubt. Der Begriff <Camp〉 wurde in den I96oer Jahren von Susan Sontag eingeführt, aber heute über Camp nachzudenken erfordert die Erkenntnis, dass sich in den letzten fünfzig Jahren zwei Dinge verändert haben: Erstens, Camp ist an die Oberfläche gelangt, wurde zum Mainstream; und zweitens, trotz seiner Assoziation mit Ausgelassenheit und Spaß
24 Vgl. Misha Kavka: Reality Television, Affect and Intimacy: Reality Matters, Basingstoke 2008. 25 Mark Andrejevic: The souissancer of Trump, in: Television \& New Media, Vol. 17, Nr. 7, 2016, 651-655, hier $6_{52}$. 
seit den I970ern, macht sich Camp gegenwärtig den negativen Affekt zu eigen und münzt ihn in ein Spektakel schlechten Benehmens um. Eine neue Form der Schamlosigkeit begleitet dieses Verhalten, das die Zivilgesellschaft als durch transgressive Performances der Wut, Verzweiflung, Frustration und sogar des Hasses transformiert begreift, die in paradoxerweise vergnüglichen Szenen televisuell inszenierter Gewalt zur Aufführung gelangen. Zwar hat es etwas Spielerisches und Befreiendes, Menschen dabei zuzuschauen, wie sie sich daneben benehmen. Darin liegt jedoch zugleich etwas Bedrohliches, das mit der vertrauten Beziehung des Fernsehens zur <realen Welt> zu tun hat - eine Beziehung, die im Realitätsfernsehen überhöht wird, indem es darauf besteht, die Kameras auf reale Menschen zu richten, die sich selbst <spielen〉 müssen. Gerade wegen seiner porösen Beziehung zur sozialen Realität gehen die transgressivsten dieser Shows weiter, als bloß die Theatralik oder Künstlichkeit des Fernsehens zu zelebrieren. Sie arbeiten systematisch daran, renitentes Verhalten in den Vordergrund $\mathrm{zu}$ rücken, es zu belohnen und sogar zu ermächtigen - und produzieren damit eine neue Unzivilisiertheit, eine unzivilisierte Gesellschaft, angetrieben von der verdichteten Dynamik der Reality-TV-Sendung. Trump, der sich mit der Missachtung von Regeln brüstet, brachte das aus dem Fernsehland mit.

Freilich sind diese Darstellungen nicht typisch für sämtliche Reality-TVSendungen. Sie beschränken sich tendenziell auf jene, wo das vorrangige <Talent> der Teilnehmer_innen in der expressiven Performance des affektiven Selbst besteht. Kritiker_innen wie Alison Hearn haben die Performance einer Widerspenstigkeit auf dem Bildschirm zu Recht als eine Form affektiver Arbeit identifiziert, wobei die Arbeit der Teilnehmer_innen darin besteht, vor der Kamera gefühlserzeugende Beziehungen einzugehen und damit Bildmaterial zu produzieren, das den Fernsehzuschauer_innen dann über die Werbetreibenden verkauft wird. Der Begriff der affektiven Arbeit lässt sich auf Michael Hardts gleichnamigen Artikel aus dem Jahre 1999 zurückführen, der seine Zusammenarbeit mit Antonio Negri aufgreift und folgende Millenniumsverlautbarung macht: «Affektive Arbeit», erklärt er, «hat im Verhältnis zu anderen Formen der Arbeit in der globalen kapitalistischen Ökonomie eine dominante Position eingenommen». ${ }^{\mathbf{2 6}}$ Die vergangenen fünfzehn Jahre scheinen Hardt und Negri Recht zu geben; wir bewegen uns nun bewusst in einem ökonomischen Paradigma, das mit «Informationen, Kommunikation, Wissen und Affekt» ${ }^{27}$ handelt; was nun den Affekt von den ersten dreien unterscheidet, ist seine Funktion als Arbeitsmittel, das die anderen als immaterielle Güter hervorbringt. In Übereinstimmung mit der These, die er zusammen mit Negri in Empire (2000) entwickelt, schreibt Hardt, dass die affektive Arbeit wegen der Ephemerität ihrer Produkte selbst immateriell sei, und präzisiert sie als «ein Gefühl der Erleichterung, des Wohlbefindens, von Zufriedenheit, Begeisterung, Leidenschaft - sogar ein Gefühl der Verbundenheit». ${ }^{\mathbf{2}}$

26 Hardt: Affective Labor, hier go.

27 Ebd., 93.

28 Ebd., 96.
Was aber passiert mit diesem Affektbegriff, der in Ton und Produktivität durchweg positiv ist, wenn sich die affektive Arbeit auf Widerspenstigkeit 
gründet und dadurch konstituiert, dass sich Leute ermächtigen und schlecht benehmen? Sich schlecht zu benehmen besitzt zweifellos Klassenimplikationen, die Trump in seiner Performance als Präsidentschaftskandidat und Präsident anzuzapfen und zu verstärken sucht. In dieser Hinsicht hat das Realitätsfernsehen einen unzivilen Aufstand der Bevölkerung befördert, der von elitären ästhetischen Formen bislang unterdrückt worden war. Ich würde jedoch argumentieren, dass uns das Fernsehen - besonders als Medium der in das politische Feld verpflanzten jouissance - dazu bringt, die Kapazitäten und Produkte affektiver Arbeit im Kontext renitenten Verhaltens, sichtbar geworden durch hyperreale Medien wie Realitätsfernsehen, Talkshows und Kabelnachrichten, neu zu denken. Obwohl diese Leute mit ihren Emotionen arbeiten, heißt das nicht zwangsläufig, dass das Ergebnis ihrer Arbeit ein immaterielles Produkt ist. Insofern als das schlechte Benehmen im Fernsehen, quer durch die sozialen Medien und in den politischen Räumen Widerhall findet, nimmt die Verhandlung dieser Affekte durchaus materielle Form an und besitzt <reale> Konsequenzen. Es geht dabei nicht einfach um ein gutes Gefühl produziert als Erleichterung oder Wohlbefinden oder gar als <positives Konzept $>$ die eigene (laute) Stimme zu nutzen, um die eigenen Interessen zu sichern; es geht vielmehr um die vergnügliche Performance eines <schlechten> Gefühls, das für Körper, die durch Gender, Sexualität, <Rasse>, Ethnizität und Klasse von hegemonialen Privilegien ausgeschlossen sind, eine Verschiebung der sozialen Regeln produziert. Hinzu kommt, dass sich das Spektrum möglicher Akteur_innen, die in dieses Fest schlechter Gefühle hineingerissen werden, beträchtlich erweitert hat, im direkten Verhältnis zum Grad, in dem der Bereich hegemonialer Privilegierung geschrumpft ist. Zur Schau gestellt wurde und wird das auf Trumps eigenen Wahlkampfveranstaltungen, wo der Faschismus seiner souveränen affektiven Performance - sprich seiner Darstellung der Souveränität des (negativen) Affekts - zu einer Frage des (lauten) Tons und der sukzessive erhöhten Taktung gerät.

Ich argumentiere nicht für die Beanspruchung schlechter Gefühle, gewiss nicht in der Form rassistischen, xenophobischen, misogynen - oder faschistischen - Hasses. In politischer Hinsicht sind Trumps Wahlkampfveranstaltungen das Gegenteil revolutionärer Rhetorik. Was sich jedoch zeigt, wenn wir unsere Aufmerksamkeit von der Rhetorik auf den Affekt verlagern, ist der affektive Thrill, der Grad an oft physischer Freude, den die Teilnehmer_innen auf Trumps Wahlkampfveranstaltungen erleben, wenn sie auf jemanden losgehen, die Regeln brechen, «sagen, wie es ist>. Es war nie Trumps Rolle, auf diesen Veranstaltungen Ideologie- oder Politikvermittlung zu betreiben, sondern vielmehr über die Modulationen seiner Stimme, Gesten und Performance ein transgressives Hochgefühl schlechten Benehmens zu ermöglichen oder gar anzustacheln - alles durch seine Rolle als Urvater des Vergnügens, als dankbarer Zuschauer kakophonischer Kämpfe, wie er es in The Apprentice getan hat. Es ist die Erkenntnis einer Allianz von Spektakel und transgressivem Thrill, die 
uns in eine andere performative und kritische Tradition führt: Camp. Anders als die politisch subversive Verspieltheit queerer Drag-Künstler_innen müssen Trumps Wahlkampfveranstaltungen allerdings als Beispiel extrem negativen Camps diskutiert werden.

\section{Camp-Mutationen}

Seit Mitte der 2oooer Jahre wurde das Realitätsfernsehen immer offener mit Künstlichkeit und übertriebenen Performances gleichgesetzt. Einerseits lenkt das vom Anspruch auf <Realität $>$ ab, den es auf Grundlage seiner dokumentarischen Ästhetik hatte, andererseits jedoch ließe sich sagen, dass diese Umarmung einer Wiederaneignung der Künstlichkeit als Anspruch auf Sichtbarkeit gleichkommt, vergleichbar mit dem, was mit dem Wort <queer> in den I98oer und I990er Jahren geschah. In der Tat, gemessen an den Normen korrekten öffentlichen Benehmens wurden Ausraster, Zickenkrieg und das übertriebene Spektakel im Fernsehen, wenn auch heterosexualisiert, zu einem neuen Gebiet queeren Verhaltens. Unter diesem Blickwinkel ist Trump eine queerende Figur, in einem viel älteren Sinne des Begriffs, zugleich throwback und backlash gegen feministische und LGBT-Politik. Gleichwohl steht er jedoch an der Spitze eines wachsenden Pathos, das sowohl Inhalt als auch Antrieb der Performativität der neuen Genres ist: «Realitätsfernsehen, Pro-Wrestling und Kabelnachrichten, besonders Fox News», die er sich zu eigen gemacht hat ${ }^{29}$ Wie ich bereits bemerkt habe, ist der Zugang zu solch einer performativen Künstlichkeit nicht gleichmäßig verteilt; wie die Reality-Programme im Rhythmus der catfights zeigen, tendiert er dazu, eine hochgradig gegenderte, rassifizierte, sexualisierte und schichtbasierte Form anzunehmen. Aber gerade das macht eine derart sichtbare, affektive Performativität zum Vehikel, das Gender, <Rasse>, Sexualität und Klasse als Orte des Machtkampfes in Erscheinung treten lässt.

Zusammengenommen suggerieren die Begriffe Künstlichkeit, Übertreibung, Pathos, Performance - fünfzig Jahre nach Susan Sontags bahnbrechendem Artikel in The Partisan Review (1964) -, dass es an der Zeit ist, uns Sontags «Anmerkungen zu Camp» erneut zuzuwenden. Camp ist allerdings nicht mehr eine spezialisierte urbane, ästhetische Formation - «eine Art Geheimkode», ${ }^{30}$ wie sie es Mitte der I96oer formulierte -, sondern eine Erlebnisweise, die in den amerikanischen Mainstream Eingang gefunden hat, von TV-Comedy und Serien über Nachrichten und das Tagesgeschehen - und nun allem voran auf der politischen Bühne. Zu fragen ist mithin, was mit der Logik und der Funktion von Camp geschieht, wenn «You bitch!»-Rufe oder das Mantra bei Trump-Veranstaltungen wie «Bau diese Mauer!» und «Sperr sie weg!» zum Kennzeichen - oder gar zum Auslöser von Vergnügen - der populären Medienkultur werden?

Sontag selbst positionierte Camp als ästhetisch, aber unpolitisch, einen Created Donald Trump. die augenzwinkernde Schönheit im Hässlichen zelebriert. In der ersten ihrer 
$5^{8}$ Anmerkungen definiert Sontag Camp als ästhetisches Phänomen - «nicht um Schönheit geht es dabei, sondern um den Grad der Kunstmäßigkeit, der Stilisierung», obwohl sie in der zweiten Anmerkung darauf besteht, dass die Erlebensweise des Camp «entpolitisiert - oder zumindest apolitisch - ist» - ein Gesichtspunkt, so offensichtlich für sie, dass er «sich von selbst» ${ }^{31}$ verstehe. Mitte der I970er Jahre war Camp jedoch hochpolitisch geworden, bestimmt von der Politik einer, wie Jack Babuscio formuliert, «Gay-Sensibilität». ${ }^{32}$ Das Ziel des schwulen, männlichen Camp, assoziiert mit Underground-DragPerformances der I970er und I980er, war die Destabilisierung von Ideologien über die Selbstverständlichkeit von Gender und Sexualität durch Performances, die weibliche und männliche Attribute an denselben Körper band. Trotz der breiten Zirkulation von Camp in den vergangenen zehn Jahren (auch dank einer anderen Realityshow, RuPaul's Drag Race, aber das ist eine andere Geschichte) blieb die Polarisierung zwischen der apolitischen Ästhetik von Kunstfertigkeit und der stilisierten Politik einer queeren Subkultur unter Praktiker_innen wie Theoretiker_innen weitgehend bestehen. Während die Sichtbarkeit des Stils und der Signaturen von Camp im Mainstream zunimmt, dient die kulturelle Ansammlung von Performances online, auf dem Bildschirm und auf der politischen Bühne dazu, den traditionellen Camp-Kanon zu entpolarisieren und in die Sprache des <neuen Normalen> zu verlängern.

Das bedeutet nicht, dass Camp seine traditionelle Verbindung zur Queerness verloren hätte, aber es deutet darauf hin, dass sich queere Sprache, Stilistik und Politik zunehmend zerstreuen und ihnen neue Funktionen zugeschrieben werden. Es gibt einerseits die Hoffnung, dass dieser Prozess des Mainstreaming der Ausdehnung der politisierten Queerness von Camp auf Identitätsgruppen dient, die traditionsgemäß außerhalb des Camp-Kanons lagen; nicht nur auf nicht schwule Gender-Identitäten, sondern auch auf eine Reihe von regionalen, ethnischen, religiösen, <Rassen >- und Klassenidentitäten, die nun die CampPerformativität nutzen, um Sichtbarkeit und Bedeutung zu beanspruchen. Auf der anderen Seite hat der Erfolg von Trumps Präsidentschaftskandidatur klargemacht, dass Camp als politisches Mittel auch jenen einst hegemonial privilegierten Gruppen - die weiße, männliche, konservative Mittelschicht im Besonderen - zur Verfügung steht, die jegliche Gemeinsamkeit mit Queer-Perspektiven leugnen würden. Trump ist selbst ein Meister des kodierten Sprechens, das die weiße, patriarchale Vorherrschaft im Gewande des Camp in die politische Arena schmuggelt - ob er garantiert, dass die Größe seiner Hände nicht bedeute, dass «etwas anderes klein sein muss», ${ }^{33}$ oder ob er twittert, dass Senatorin Kirsten Gillibrand «vor nicht allzu langer Zeit» für eine Wahlkampfspende von ihm «alles getan hätte» (I I. Dezember 20I7). Dieses kodierte Sprechen in CampManier appelliert an den Sinn weißer Entrechtung; es gibt den Ton seiner Performances auf den Kundgebungen vor, ermöglicht die Selbstidentifizierung von Trumps Anhänger_innen und - im erbittertsten Kampf überhaupt - konsolidiert und entblößt die Struktur weißer, patriarchaler Überlegenheit.
31 Sontag: Notes on Camp, hier Punkt 1 u. Punkt 2.

32 Jack Babuscio: Camp and the Gay Sensibility, in: David Bergman (Hg.): Camp Grounds: Style and Homosexuality, Amherst 1993, 19-38.

33 Fox-News-Debatte, 3.3.2016. 
Das ist mithin genau der Punkt: Das Mainstreaming des Camp hat die übertriebene emotionale, queere Performance Gruppen zugänglich gemacht, die diese Performances unter den Marginalisierten ironischerweise überhaupt erst notwendig machten, weil sie selbst das hegemoniale Ideal verkörperten. In anderen Worten: Wenn Camp das neue Normale ist, dann nur, weil das $<$ Mittelamerika> der Fly-Over-Staaten die neue Unterschicht ist und nun seine eigene Bühne einfordert - von Trumps permanenten Kundgebungen bis hin zu den zahlreichen Bildschirmen des populistischen Fernsehens -, um in laute, raue und übermäßig wütende Performances einzustimmen und sich damit Gehör zu verschaffen. Was dabei wichtig ist: Inmitten dieser übertriebenen Artikulationen der neuerdings Marginalisierten hat sich die traditionelle Theatralität des Camp von etwas, das Sontag als «Erfahrung des Unterengagements, der Gleichgültigkeit» ${ }^{34}$ bezeichnet, zu etwas verschoben, das ich als Modus eines Mainstream-Überengagements bezeichnen würde, sprich: Performances, die negativen Camp mit der Fähigkeit ausstatten, affektive Forderungen sichtbar, hörbar und erkennbar zu machen. Ästhetischer Camp der i $960 e r$ Jahre sagte: $<$ Wir hüten uns davor, den normativen Geschmack ernst zu nehmen〉; schwuler männlicher Camp der I970er/8oer sagte: <Wir hüten uns davor, normative Geschlechter und Sexualität ernst zu nehmen〉; Mainstream-Camp unserer Tage sagt: <Wir hüten uns davor, die normative Politik ernst zu nehmen, weil wir selbst die Norm waren $>$.

\section{Sassy Trump}

Wie ich bereits erörtert habe, ist der Trump des The-Apprentice-Boardrooms das Herzstück des obszönen Vergnügens; in Žižeks Begriffen Vater Fouissance, der die Kastration verweigert. ${ }^{35}$ Aber Trumps Narzissmus ist dergestalt, dass er in diesem Kurzschluss zwischen «unreflektierter Fantasie» und «kluger Skepsis ${ }^{36}$ beide Rollen spielen kann; er kann sowohl der obszöne Vater sein, der genussvoll zuschaut, wie sich jemand schlecht benimmt, als auch derjenige, der sich zum Vergnügen der Zuschauer_innen selbst daneben benimmt. Er kann, in anderen Worten, der «anzüglich grinsende, triebhafte Übermensch $\gg^{37}$ und der Camp-Performer sein, der mit Vergnügen betrachtet und sich daran erfreut, selbst betrachtet zu werden.

Vermutlich war Trump bereits in den I980er Jahren Camp: durch seine liebgewonnene Kombination von hohen Kosten und niederem Geschmack und seine Vorliebe für sorgfältig inszenierte Privataufnahmen mit Ivana - beide formell gekleidet in Anzug und Satin, an einem ausladenden Treppenaufgang am Faulenzen. Ironischerweise ist Trumps Fähigkeit zu Camp mehr als ober-

34 Sontag: Notes on Camp, hier Punkt 44. 35 Vgl. Andrejevic: The Jouissances of Trump, $65_{2}$.

36 Ebd.

37 Ebd. flächlich. Sie hat mit der Art und Weise zu tun, wie er Hyper-Maskulinität als Kunstgriff, als etwas durch Gesten, Posen und Präsentationsstile, die alles andere als männlich sind, Aufgesetztes oder <Angezogenes> darstellt. Man beachte etwa sein Beharren darauf, dass sein Haar, das fast ebenso schwungvoll ist 
wie seine Treppen, <real> ist, ein Refrain, der zurückgeht auf seine ApprenticeTage. Oder die ernsthafte, aber offen übertriebene Art, wie er für seine Marke, seine Hotels, seine Präsidentschaft, sein Amerika wirbt (falls Sie sich gefragt haben sollten - es sind immer <die besten>). Er ist in vielerlei Hinsicht der theatralischste US-Präsident in der jüngeren Geschichte, besonders was die Performance seiner Maskulinität anbelangt: Denken Sie an das Problem mit den <kleinen Händen>, den offenkundig phallisch konnotierten Abwurf der MOAB (mother of all bombs) auf einen Höhlenkomplex der ISIS in Afghanistan, die Auseinandersetzungen mit National-Football-League-Spielern, die während der Nationalhymne <niederknien>, oder eine beliebige Auswahl aus den fast täglichen Beispielen. Wie jeder gute Camp-Performer scheint Trump zu wissen, dass es nicht darum geht, was man <da unten〉 hat oder nicht, sondern darum, wie man es präsentiert.

Nirgendwo wird diese inhärente Campyness auf glänzendere Weise freigelegt als in der Sassy-Trump-Serie, einem Set von YouTube-Videos des britischen Komikers Peter Serafinowicz, die erstmals auftauchten, als Trump Wahlkampf für die republikanische Nominierung betrieb, und sich bis in seine Präsidentschaft fortsetzen. ${ }^{38}$ Serafinowicz nimmt in dieser Serie Clips von Trumps Performances auf Kundgebungen, Debatten und in präsidialen Reden und legt seine eigene Stimme über die Tonspur. Damit ersetzt er Trumps Stimme mit einer feminisierten Camp-Männerstimme; alles andere jedoch gehört dem Original: Die Worte sind jene, die Trump tatsächlich sprach, passend zu den Gesten, Bewegungen, Mimik und Pausen, wie er sie vollzog. Was an dieser Serie überrascht wie auch auf komische Weise Aufschluss gibt, ist, wie sehr die Performance Sinn ergibt. Es scheint vollkommen real - so real wie Trumps eigene Haare -, gerade weil die feminisierte Stimme offenlegt, dass die Worte, Gesten und das Mienenspiel schon immer Teil einer Camp-Performance waren; tatsächlich verleiht Serafinowicz dem Camp-Ton, der von jeher Teil von Trumps Performance war, den angemessenen Tonfall. Die Finger, die weit gespreizt werden, kurz bevor die Hände zur Betonung die Luft durchschneiden; die hochgezogenen Augenbrauen, die bewusst für das TV-Kamera-Close-up verharren; die Pausen zwischen den Worten, die verschwindenden Sätze, die Rhetorik, die Lücken lässt für das Gelächter, Jubel und Applaus des Publikums, sobald die Doppeldeutigkeiten durchschaut sind - all das gehört nicht nur zum Arsenal eines Showmans, sondern auch zu dem eines Camp-Performers, der lediglich einen Dreh im Tonfall benötigt, um als queer geoutet zu werden. Die Sassy-Trump-Serie mag das Ausmaß offenlegen, zu dem Trump bereits Camp ist. Aufgrund unserer Erwartungen, dass Männer mit seinem Status, Vermögen und sexuellen Neigungen zweifellos maskulin zu sein haben, neigen wir jedoch dazu, es nicht zu sehen oder zu hören. Aber natürlich sind, wie ich herausgestellt habe, die Requisiten, die er benötigt, um seinen Status, Reichtum und Neigungen etc. zu zeigen und zu bewahren, selbst Camp-Objekte, bis hin zu den Ex-Models, die regelmäßig seinen Arm dekorierten und mitunter seine Ehefrauen wurden.
38 Vgl. etwa die «Best of Kompilation veröffentlich auf YouTube von Papa Woody: The Best of Peter Serafinowicz's Sassy Trump, www.youtube.com/ watch? $=d 76 \mathrm{~g}$ Culct $4 \mathrm{~A}$, dort datiert 14.8.2016, gesehen am 18.2.2018. 
In dieser campy Persona kommt alles zusammen: der Narzissmus, die Empfindlichkeit gegenüber Kritik, die Beziehung zu Putin (selbst mit einer Tendenz zu übertriebenen, oberkörperfreien Performances der Männlichkeit) und nicht zuletzt all der Hass auf jene Medien, die sich weigern, ihn im besten Lichte zu zeigen. Serafinowicz' Version des <real_Donald_Trump> ist freilich selbst Camp, gehört jedoch zu einem älteren Camp-Modus, einem politisierten schwulen Camp, der Humor generiert, gerade weil er so plausibel daherkommt. Der Genius dieses Humors liegt darin, dass er Trumps Worte - die sehr wenig semantischen Gehalt bieten - vergessen macht und stattdessen unsere Aufmerksamkeit auf die Performance als affektive Modulation lenkt, wo Trumps Klugheit und seine geschmeidige Integration in die politische Gegenwart liegen. Trump als Camp zu begreifen heißt zu verstehen, dass wir, statt über Ideologie, über Affekt sprechen müssen und dass wir uns, statt auf Rhetorik, auf Performance konzentrieren müssen. Das soll nicht heißen, dass es keinerlei Politik des Affekts gibt, sondern, dass in Trumps Fall politische Ideologie seiner wissenden Performance des negativen Affekts entspringt und nicht umgekehrt.

Diese Erkenntnis ist wesentlich für die ältere Form des Camp, die von Serafinowicz mobilisiert wird und die, wie die wiederkehrende Popularität der Late-Night-TV-Satiriker_innen (insbesondere Stephen Colbert, Trevor Noah, Samantha Bee, Seth Meyers), ein effektives Mittel geworden ist, um die Wirkung von negativem politischen Camp zu unterlaufen und zu queeren. Diese Queerness ist, weil sie sich eines produktiven Aspekts der affektiven Unberechenbarkeit bedient, nicht zu unterschätzen. Das Problem allerdings hat mit der bereits erwähnten Dopplung zu tun, mit deren Hilfe Trump als Vater fouissance - eine vom Realitätsfernsehen erlernte Performance - sowohl das Subjekt wie auch das Objekt des Vergnügens sein kann. Als Subjekt des Vergnügens lehnt er sich zurück und beobachtet die emotionalen Performances der Massen, die er selbst angestachelt hat, indem er das gerechte Gefühl der weißen, patriarchalen Überlegenheit in das ironische Vergnügen der Camp-Performance kleidete. In diesem Kampf zwischen subversivem Camp (Serafinowicz) und rassistischem Camp (Trump), in dem beide Seiten das Fernsehen als Hauptkomponente ihrer affektiven Bewaffnung nutzen, lässt sich schwer sagen, wer schließlich am lautesten schreit. Recht klar ist jedoch, dass es kein guter Zeitpunkt ist, sich für die Taktik des Apprentice-Kandidaten Andy zu entscheiden und zu schweigen.

Indem Camp von einer subversiven Underground-Politik zur Oberflächenmanifestation mutierte, die im Bereich der Staatsführung zum Einsatz kommt, erfolgte diese Verschiebung vor allem mithilfe des Fernsehens, im Besonderen des Realitätsfernsehens. Schließlich ist es Realitätsfernsehen, das die veränderten Lebensbedingungen der Menschen mit der affektiven Arbeit schlechten Benehmens mischt, um eine zumindest phantasmatische Selbstbestimmung zu ermöglichen. Ich denke, es lässt sich unmöglich sagen, ob Trump Ursache oder Wirkung hiervon ist; es geht darum, sich auf die affektive Realität 
zu konzentrieren, weil sie die Logik der Kausalität, der Intention, des Resultats durchbricht, so wie es Trump mit seiner Rolle in zehn Jahren ApprenticeBoardroom-Raufereien vermittelte. Nichtsdestotrotz ist Trump der zentrale Signifikant für diese Verschiebung von Underground- zu <Overground〉-Camp, die sich über genau jene dreißig Jahre erstreckt, in denen Trump sich vom Immobilienmagnaten zum Reality-Star zum Reality-TV-Präsidenten emporarbeitete. Besonders in den letzten fünfzehn Jahren lässt sich Trumps Bewegung von der Position eines passiven Beobachters von transgressivem Verhalten (in The Apprentice), dessen Präsenz das Spektakel rechtfertigt und sicherstellt, über seine Rolle als Moderator und Anstifter negativen Camps (auf Wahlkampfveranstaltungen) bis zum Befehlshaber eines neuen Camp-Modus als dessen selbsternanntes Oberhaupt verfolgen. Jeden Schritt begleitete eine Intensivierung sowohl des transgressiven Thrills wie auch der ernsten Bedrohung für das soziale und politische Establishment, das sich immer noch der Aufklärung und demokratischer Werte rühmt. Zugleich haben jedoch, wie ich erörtert habe, Camp und Affekt Wertehierarchien, Kausallogiken und binäre Unterschiede zwischen Thrill und Bedrohung durcheinandergebracht. Es mag vielleicht eine Fantasie der Linken sein, aber je näher Trump dem Ziel kommt, der Kommandant des Camp zu sein, desto größer wird das Potenzial der Rechtsaußenpolitik, die sich wie Wollmäuse an seinen Erfolg geheftet hat, von der Campyness seiner eigenen Performance untergraben zu werden.

Aus dem Englischen von Stefan Borsos 


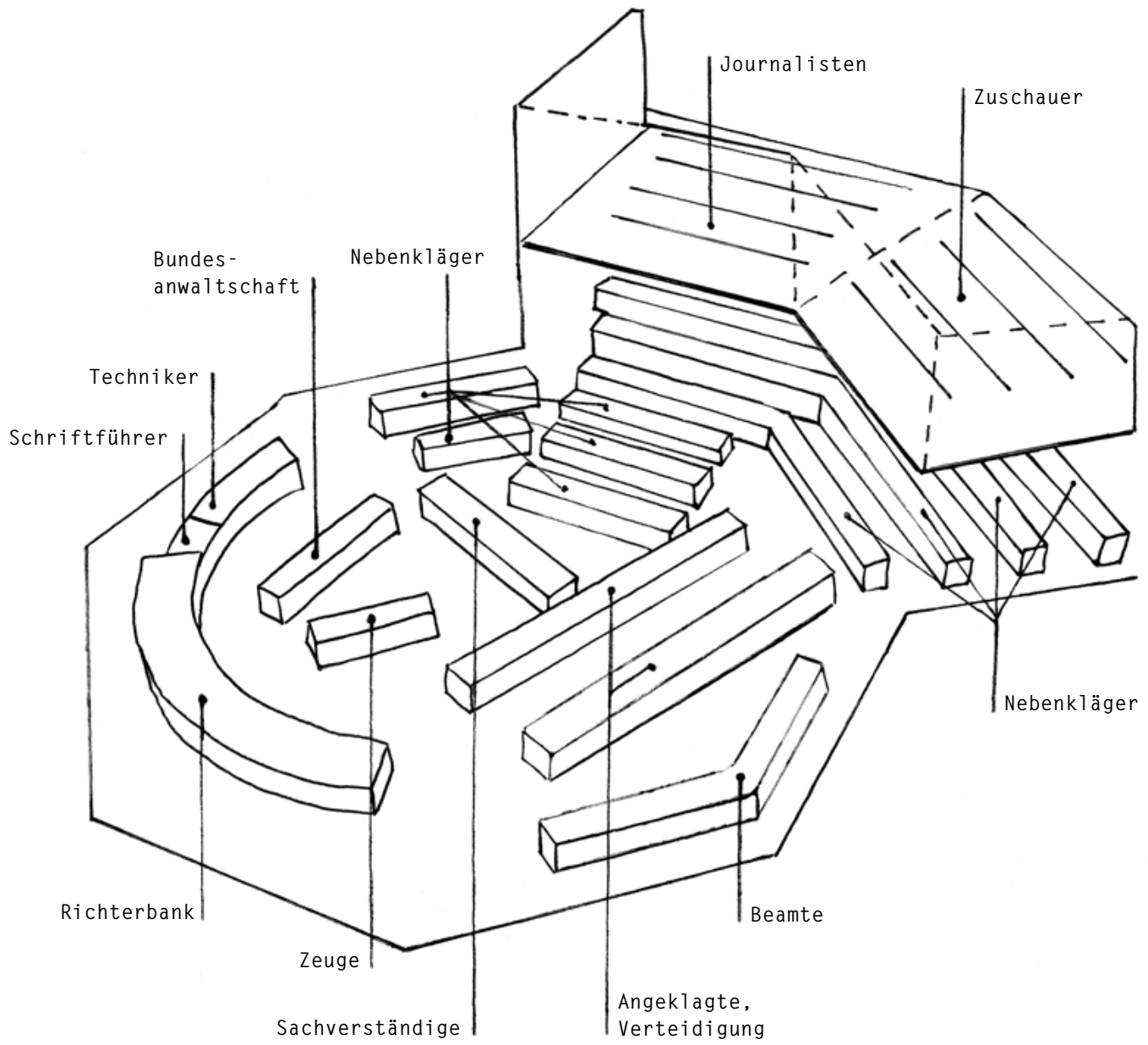

Abb. 1 Gerichtssaal ror im Oberlandesgericht in der Nymphenburger Straße I6 in München. Tische und Stühle z. B. für die Bundesanwaltschaft und Schriftführer_innen stehen nicht mehr exakt so wie abgebildet. Im Juli bzw. November 2015 wählte die Angeklagte B. Zschäpe noch zwei Verteidiger hinzu (jetzt hat sie fünf Verteidiger_innen).

Angeklagte und Verteidigung nehmen nun insgesamt drei Tisch bzw. Stuhlreihen in Anspruch. 